\title{
Normal form analysis of integrable Hamiltonian systems
}

\author{
R.C. Miranda Filho and R.F.S. Andrade \\ Instituto de Física, Universidade Federal da Bahia, 40210 Salvador, Brazil
}

Received 28 May 1990; revised manuscript received 20 September 1990; accepted for publication 28 November 1990 Communicated by D.D. Holm

\begin{abstract}
Normal form transformations are used to analyse two integrable cases of the generalized Hénon-Heiles Hamiltonian. The purpose of this Letter is to explore the explicit construction of transformations which give rise to the correct second integral for non-trivial integrable systems. For both investigated cases it is found that just one such transformation exists and that it cannot be constructed without the previous knowledge of the second integral.
\end{abstract}

There has been a series of recent works on the normal form transformations (NFTs) stressing their potential as a tool to explore non-linear differential equation systems [1-5]. One main aspect emphasized therein is the potential usefulness of the NFT to the exploration of non-integrable dynamical systems. Several non-integrable prototypes were considered. Most of the successes and limitations of the method were discussed based on such studies.

On the other hand, less effort has been devoted to the NFTs of integrable systems. An important result on this subject was derived by Russmann [6], who proved the existence of a convergent NFT series for any integrable non-resonant Hamiltonian system with two degrees of freedom. However, Russmann's work addresses only the existence of the NFT: the process of obtaining this explicit transformation and the question concerning its uniqueness are left open.

In this Letter we use NFTs to treat resonant integrable Hamiltonian systems, which are not covered by Russmann's results. Our attention is focused on the explicit construction of finite order NFT truncations for these models rather than proving series convergence. However, interesting insights about convergence and uniqueness questions arise within the developed procedure when a comparison is drawn between the exact integrals of motion and the NFT integrals. Based on this comparison process we come to the following conclusions, which may also be valid for other similar models: (i) there exists just one convergent canonical transformation; (ii) this particular transformation cannot be constructed without the knowledge of the exact integrals.

It is well known that the NFTs of Hamiltonian systems may be obtained both with the near identity transformation (NIT) [7] and the Birkhoff-Gustavson normal form transformation (BGNFT) [8]. Both schemes lead to the same equations of motion for the transformed system, though the relations between the new and old coordinates are different for finite order approximations. Throughout this work we will use the NIT method to reduce the systems to their NFTs.

Let us recall that certain generalizations of nonintegrable systems lead to integrable cases [9-11]. They have been identified after the explicit construction of integrals of motion other than the energy or by looking for conditions in the parameter space which satisfy the Painlevé property. These cases do allow for rigorous integrals of motion, expressable as algebraic forms of some degree. So we will consider, according to ref. [11], the following parametrization of the Hénon-Heiles model:

$H=\frac{1}{2}\left(p_{1}^{2}+p_{2}^{2}+A q_{1}^{2}+B q_{2}^{2}\right)+q_{1}^{2} q_{2}-\frac{1}{3} \epsilon q_{2}^{3}$,

where three integrable cases may be identified:
(a) $\epsilon=-1, A=B$,
(b) $\epsilon=-6 \quad \forall A, B$,
(c) $\epsilon=-16, \quad B=16 A$. 
The following integrals are respectively valid for the cases above:

$$
\begin{aligned}
G_{\mathrm{a}} & =p_{1} p_{2}+\frac{1}{3} q_{1}+q_{1} q_{2}^{3}+A q_{1} q_{2}, \\
G_{\mathrm{b}} & =q_{1}^{4}+4 q_{1}^{2} q_{2}^{2}-4 p_{1}\left(p_{1} q_{2}-p_{2} q_{1}\right)+4 A q_{1}^{2} q_{2} \\
& +(4 A-B)\left(p_{1}^{2}+A q_{1}^{2}\right), \\
G_{\mathrm{c}} & =3 p_{1}^{4}+6\left(A+2 q_{2}\right) q_{1}^{2} p_{1}^{2}-4 q_{1}^{3} p_{1} p_{2} \\
& -4 q_{1}^{4}\left(A q_{12}+q_{1}^{2}\right)+3 A^{2} q_{1}^{4}-\frac{2}{3} q_{1}^{6} .
\end{aligned}
$$

Hereafter we will carry out an NFT analysis of the integrable cases (a) and (b). After that we compare the obtained NFT integrals of motion with the $G_{\mathrm{a}}$ and $G_{\mathrm{b}}$ given by ( $\left.3 \mathrm{a}\right)$ and ( $\left.3 \mathrm{~b}\right)$. Our main results follow then after this comparison. Moreover, since (a) requires only $A=B$ and (b) makes no restriction on the value of these parameters we reduce the algebraic work by requiring $A=B=1$ and leaving $\epsilon$ as free parameter.

The use of the NIT in connection with NFTs has been explored in a number of recent articles [1-5]. So we will briefly outline the above scheme here, while the reader should refer to the quoted works for details [3]. For the sake of simplicity in the derivation of the NIT one first considers a coordinate transformation by requiring that the linear part of the system should be in a diagonal form. In the present case this is done by defining

$$
\text { (a) } x_{2 j}=\frac{1}{\sqrt{2}}\left(q_{j}+\mathrm{i} p_{j}\right), \quad \text { (b) } x_{2 j-1}=\mathrm{i} x_{2 j}^{*} \text {. }
$$

The above transformation is canonical, which is quite important for the rest of the discussion in this Letter. The equations of motion in terms of $\left\{x_{i}\right\}$ are

$$
\begin{aligned}
& x_{1}=\mathrm{i} x_{1}+\frac{1}{\sqrt{2}}\left(x_{1}+\mathrm{i} x_{2}\right)\left(x_{3}+\mathrm{i} x_{4}\right), \\
& x_{3}=\mathrm{i} x_{3}-\frac{1}{\sqrt{2}}\left[\epsilon\left(x_{3}+\mathrm{i} x_{4}\right)^{2}-\left(x_{1}+\mathrm{i} x_{2}\right)^{2}\right] .
\end{aligned}
$$

We point out that (5) belongs to the class of nonlinear systems with highest non-linearities of second degree, whose general structure is

$\dot{x}_{i}=\lambda_{i} x_{i}+\sum_{k, l} A_{i, k l} x_{k} x_{l}$,

where $\lambda_{i}= \pm \mathrm{i}$ and the coefficients $A_{i, k l}$ can easily be read from (5). The sth order NIT from $\left\{x_{i}\right\}$ to new coordinates $\left\{y_{i}\right\}$ is given by $x_{i}=y_{i}+g_{i}(y)=y_{i}+\sum_{|m| \geqslant 2}^{s} \alpha_{i}(m) y^{m}$,

where $m=\left(m_{1}, m_{2}, m_{3}, m_{4}\right)$ and we introduce the multinominal notation. The $\alpha_{i}(m)$ are so chosen as to eliminate all non-linear terms. This can be accomplished for all but resonant terms, i.e., those which satisfy the resonance condition

$(m, \lambda)-\lambda_{i}=0$,

where $\lambda$ is the vector formed by the eigenvalues of the linear part of the original system and $($,$) in-$ dicates the internal product. The transformed system now reads

$\dot{y}_{i}=\lambda_{i} y_{i}+\sum_{|m| \geqslant 2}^{s} \beta_{i}(m) y^{m}+\eta_{i}(y)$,

where the prime indicates that the sum is restricted to the resonant terms and $\eta_{i}(y)$ denotes the so-called rest term $\sim|y|^{s+1}$. Its presence in (9) asserts that the transformed system is fully equivalent to the original one. However, within the NFT we normally neglect the $\eta_{i}$ and consider successively higher order approximate systems, which are formed by the first two terms on the r.h.s. of (9). These truncations are referred to as an sth order approximation.

For systems like (6) the $\alpha$ 's and $\beta$ 's can be given at once as:

(a) non-resonant cases:

$\alpha_{i}(m)=\frac{\gamma_{i}(m)}{(m, \lambda)-\lambda_{i}}, \quad \beta_{i}(m)=0$,

(b) resonant cases:

$\alpha_{i}(m)=$ arbitrary, $\quad \beta_{i}(m)=\gamma_{i}(m)$,

where $\gamma_{i}(m)$ is given by

$$
\begin{aligned}
& \gamma_{i}(m)=\sum_{k, l} A_{i, k l}\left(\delta\left(m, \mu_{k}+\mu_{l}\right)+2 \alpha_{l}\left(m-\mu_{k}\right)\right. \\
& \left.+\sum_{\left|m^{\prime}\right| \geqslant 2}^{|m|-3} \alpha_{k}\left(m^{\prime}\right) \alpha_{l}\left(m-m^{\prime}\right)\right) \\
& \quad-\sum_{k} \sum_{\left|m^{\prime}\right| \geqslant 2}^{|m|-2} \beta_{k}\left(m^{\prime}\right) \alpha_{i}\left(m-m^{\prime}+\mu_{k}\right) \\
& \quad \times\left(m_{k}-m_{k}^{\prime}+1\right) .
\end{aligned}
$$

In (11) $\mu_{k}$ represents the unit vector in the $k$ th direction. 
For practical purposes the application of the program just sketched in eqs. (6)-(11) can be pursued without computer help only when $s \leqslant 4$. From this order up the recurrence relations become rather untractable. A computer is then required either for the numerical evaluation of the coefficients or for the algebraic manipulation of the polynomial relations. We developed a system which gives both the $\alpha_{i}(m)$ and $\beta_{i}(m)$ as a polynomial function of $\epsilon$ as well as their numerical value for a fixed value of $\epsilon$. This program works fully automatic, except for the determination of the values of the arbitrary resonant $\alpha$ 's.

The resonant $\alpha$ 's may take arbitrary values. This reflects the fact that the NIT of resonant models gives rise not to a single NFT but to a whole family of transformations. This multiplicity of NFTs is not characteristic of its construction with the help of the NIT, but it is a feature also present in the BGNFT. One major difference between these two schemes refers to the fact that the whole family of BGNFTs consists of canonical transformations, for they are constructed from a family of generating functions of old and new variables.

On the other hand the method of NIT does not require that the transformations should be canonical. However, it is well known that a necessary and sufficient condition for a transformation to be canonical is that the Poisson brackets between old and new variables,

$$
\left[x_{i}, x_{j}\right]=\sum_{k=1}^{N}\left(\frac{\partial x_{i}}{\partial y_{2 k}-1} \frac{\partial x_{j}}{\partial y_{2 k}}-\frac{\partial x_{i}}{\partial y_{2 k}} \frac{\partial x_{j}}{\partial y_{2 k-1}}\right),
$$

take their proper values 0 or 1 [12]. Therefore it is natural to ask what their values are in the present case. We identify two important features in the evaluation of (12):

(i) Polynomial transformations like (7) cannot be exactly canonical. Indeed, the evaluation of (12) for an sth order NFT shows that it entails terms of order up to $2 s-2$, though only those up to order $s-1$ are complete, i.e., they will not be affected when the order of the NFT is increased from $s$ to $s+1$. The evaluation of (12) shows that, as long as no resonant $\alpha$ appears, it differs from the required values for canonical transformation by terms of order higher than $s-1$.

(ii) When the first resonant $\alpha$ 's appear in (12) for $s=s^{*}$, they will influence only the terms of order greater than $s^{*}-2$. If we demand that the proper values 0 or 1 be kept for order $s^{*}-1$ we obtain a linear system of equations for the resonant $\alpha$ 's. The number of equations equals that of the unknowns and its solution will restore the situation discussed above. From now on we refer to the canonical character of the NIT, but we implicitly acknowledge that the correct values of the Poisson bracket are obtained under restriction to terms of order up to $s-1$.

For both cases (a) and (b) the corresponding systems are underdetermined. They admit families of canonical NFTs, yet smaller than the full original sets. This multiplicity is much like the one obtained for the BGNFTs.

For the model (1) the first resonances are observed when $s=3$. The resonant $\alpha$ 's, whose values have been established by the Poisson bracket condition, are

$\alpha_{1}(1,0,1,1)=\alpha_{3}(1,1,1,0)=\frac{16}{9} \mathrm{i}$,

$\alpha_{1}(2,1,0,0)=\frac{8}{9} i$,

$\alpha_{3}(0,0,2,1)=\frac{8}{9} \mathrm{i} \epsilon^{2}$,

$\alpha_{1}(0,1,2,0)+\alpha_{3}(2,0,0,1)=-\frac{16}{9} i \epsilon$.

The last equation shows explicitly the fact that a oneparameter family of canonical transformations is indeed available.

After the determination of the resonant $\alpha$ 's we can proceed with the comparison of the integrals of motion (3a) and (3b) with those obtained within the NFT. In the present case it turns out that the two integrals of motion within the NFT are
(a) $H_{\mathrm{NF}}$,
(b) $I_{1}=y_{1} y_{2}+y_{3} y_{4}$.

$H_{\mathrm{NF}}$ is a constant of motion for it is just the original $H$ written in terms of the new canonical variables. The other expression has been shown to be a constant of motion for the BGNFT and it is straightforward to show that the same result holds in the case of the NITs. Moreover since only two independent integrals exist, it is possible to express $H_{\mathrm{NF}}$ in terms of the simple expressions $I_{1}$ and $I_{2}$. The form of the latter is not universal as that of $I_{1}$ and depends upon the case we are considering. We get respectively
(a) $I_{2 . \mathrm{a}}=y_{1} y_{4}+y_{2} y_{3}$,
(b) $I_{2, \mathrm{~b}}=y_{1} y_{2}$.

The comparison between the two results is made by 
expressing the $G$ as functions of the new coordinates $y$. For this purpose we insert (6) into $(3 a, b)$. Here again we face the fact that comparisons only make sense if all terms of the same order are taken into account. In the case of the $G$ given in (3) the terms up to order $s+1$ are complete. So we call $G_{s+1}(y)$ the restriction of $G(x(y))$ to that order.

The next step is to gather the terms so obtained in order to express the $G_{s+1}$ in terms of $I_{1}$ and $I_{2}$. In both cases we investigated we found that, to lowest order in the power of $y$ where resonant terms appear $(s=3), G_{4}$ can only be written in terms of $I_{1}$ and $I_{2}$ if supplementary conditions are imposed on the $\alpha$ 's in (13):

(a) $\alpha_{1}(0,1,2,0)=\alpha_{3}(2,0,0,1)$,

(b) $\alpha_{1}(0,1,2,0)=8 \mathrm{i}$.

If values other than those satisfying (16) are given to $\alpha_{1}(0,1,2,0)$ and $\alpha_{3}(2,0,0,1)$ (which is clearly allowed within the canonical NFTs) we find that neither $G_{4}(y)$ is a constant of motion nor can it be corrected in higher order approximations by the inclusion of other terms.

Conditions (16) are a very compact form to condense the main results of this Letter. They can be viewed and discussed according to two distinct points of view. The first one concerns the fact that lowest order terms of the second integral $G(x)$ can be reproduced within the NFT. We may repeat the procedure for the higher order contributions and construct one particular NFT which reproduces $G$ in any order. This result is in accordance with Russmann's results, for a failure of such a reproduction would be contradictory to the existence of a convergent series.

The second point of view relates to other features which came to light in the developed procedure. We conjecture that they may be of relevance for the theory of NFTs of integrable resonant models. First, we note that (16) points in the direction of the uniqueness of the convergent NFT. Indeed, unlike relation
(13), which refers to necessary and sufficient conditions for the canonical character, (16) no longer admits a one-parameter family of NFTs. Second, we observe that the unique solutions (16) do not violate the Poisson bracket, i.e., the particular transformation which recovers the second integral is necessarily canonical. Finally, a third and most important constraint introduced into the constructed NFTs refers to the fact that solution (16) could only be found after explicit knowledge of the form of the second integral, i.e. we could not identify any limiting condition within the NIT or NFT which restricts the values of the $\alpha$ 's to those in (16). If this happens to be a general property of NFTs of integrable resonant systems, it will reduce its power to analyse such a subset of dynamical systems.

These different aspects coming from the implemented procedure point toward further investigations on this subject.

\section{References}

[1] P.H. Coullet, C. Elphick and E. Tirapegui, Phys. Lett. A 111 (1985) 277.

[2] M.K. Ali, W.R. Wood and J.S. Devit, J. Math. Phys. 27 (1986) 1806.

[3] J. Pade, A. Rauh and G. Tsarouhas, Physica D 29 (1987) 236.

[4] A. Rauh and J. Pade, preprint.

[5] A. Rauh and R.F.S. Andrade and Ch. Kougias, in: Proc. Conf. on Singular behaviour and non-linear dynamics, Samos, Greece, 1988 (World Scientific, Singapore, 1989).

[6] H. Russmann, Math. Ann. 154 (1964) 285.

[7] V.I. Arnold, Geometrical methods in the theory of ordinary differential equations (Springer, Berlin, 1983).

[8] F.G. Gustavson, Astron. J. 71 ( 1966 ) 670.

[9] Y. Aizawa and N. Saito, J. Phys. Soc. Japan 32 (1972) 1636.

[10] T. Bountis, H. Segur and F. Vivaldi, Phys. Rev. A 25 (1982) 1257.

[11] B. Grammaticos, B. Dorizzi and R. Padjen, Phys. Lett. A 89 (1982) 111

[12] H. Goldstein, Classical mechanics (Addison-Wesley, Reading, MA, 1956). 